\title{
Ultrasonic synthesis and characterization of 2D and 3D metal- organic frameworks and their application in the oxidative amidation reaction
}

\author{
Sepideh Bagheri, Farzane Pazoki and Akbar Heydari ${ }^{1}$ \\ Chemistry Department, Tarbiat Modares University, Tehran, PO Box: 14155-4838, Iran \\ Email: heydar_a@modares.ac.ir
}

${ }^{1}$ Corresponding author: HEYDAR_A@modares.ac.ir 
1 N-Cyclohexylnicotinamide: ${ }^{1}$ White solid; m.p. $149-151{ }^{\circ} \mathrm{C} ;{ }^{1} \mathrm{H}$ NMR $(500 \mathrm{MHz}, \mathrm{CDCl} 3) \delta$ $8.94(\mathrm{dd}, 1 \mathrm{H}), 8.71(\mathrm{dd}, 1 \mathrm{H}), 8.12-8.08(\mathrm{~m}, 1 \mathrm{H}), 7.37$ (ddd, 1H), $6.06(\mathrm{~d}, 1 \mathrm{H}), 4.04-3.94(\mathrm{~m}, 1 \mathrm{H})$, $2.09-2.00(\mathrm{~m}, 2 \mathrm{H}), 1.82-1.62(\mathrm{~m}, 3 \mathrm{H}), 1.50-1.37(\mathrm{~m}, 2 \mathrm{H}), 1.32-1.15(\mathrm{~m}, 3 \mathrm{H})$<smiles>O=C(NC1CCCCC1)c1cccnc1</smiles>

2 N-cyclohexylcyclohexanecarboxamide: ${ }^{2}$ White solid; m.p. $171-172{ }^{\circ} \mathrm{C},{ }^{1} \mathrm{H}$ NMR $(500 \mathrm{MHz}$, $\mathrm{CDCl} 3) \delta 5.31(\mathrm{~s}, 1 \mathrm{H}), 3.80-3.71(\mathrm{~m}, 1 \mathrm{H}), 2.02(\mathrm{tt}, 1 \mathrm{H}), 1.98-1.56(\mathrm{~m}, 9 \mathrm{H}), 1.461 .04(\mathrm{~m}, 11 \mathrm{H})$.<smiles>O=C(NC1CCCCC1)C1CCCCC1</smiles>

3 N-cyclohexylbenzamide: ${ }^{3}$ White solid; m.p. $151-152{ }^{\circ} \mathrm{C},{ }^{1} \mathrm{H}$ NMR $(500 \mathrm{MHz}, \mathrm{CDCl} 3) \delta 7.77$ - $7.73(\mathrm{~m}, 2 \mathrm{H}), 7.51-7.46(\mathrm{~m}, 1 \mathrm{H}), 7.45-740(\mathrm{~m}, 2 \mathrm{H}), 4.01-3.94(\mathrm{~m}, 1 \mathrm{H}), 1.99-1.88(\mathrm{~m}$, $1 \mathrm{H}), 1.61-1.81(\mathrm{~m}, 3 \mathrm{H}), 1.48-1.62(\mathrm{~m}, 2 \mathrm{H}), 1.45-1.34(\mathrm{~m}, 4 \mathrm{H})$.<smiles>O=C(NC1CCCCC1)c1ccccc1</smiles>

4 N-cyclohexyl-4-methylbenzamide: ${ }^{3}$ White solid; m.p. $150-152{ }^{\circ} \mathrm{C},{ }^{1} \mathrm{H}$ NMR $(500 \mathrm{MHz}$, $\mathrm{CDCl} 3) \delta 7.69-7.60(\mathrm{~m}, 2 \mathrm{H}), 6.69-6.61(\mathrm{~m}, 2 \mathrm{H}), 4.01-3.91(\mathrm{~m}, 1 \mathrm{H}), 3.01(\mathrm{~s}$, 3H), $2.01(\mathrm{~m}, 2 \mathrm{H}), 1.79-1.59(\mathrm{~m}, 3 \mathrm{H}), 1.48-1.36(\mathrm{~m}, 2 \mathrm{H}), 1.27-1.14(\mathrm{~m}, 3 \mathrm{H})$.<smiles>Cc1ccc(C(=O)NC2CCCCC2)cc1</smiles> 
5 N-(o-tolyl)butyramide): ${ }^{4}$ Yellow oil, mass spectroscopy: 177.12, found: 177.0.<smiles>CCCC(=O)Nc1ccccc1C</smiles>

6 (N-phenylbutyramide): ${ }^{4}$ Light yellow oil, mass spectroscopy: 193.246, found: 193.0.<smiles>CCCC(=O)Nc1ccccc1OC</smiles>

7 N-(4-methoxyphenyl)propionamide : ${ }^{4}$ Yellow oil, mass spectroscopy: 193.11, found: 193.0.<smiles>CCCC(=O)Nc1ccc(OC)cc1</smiles>

8 N-phenylbenzamide: ${ }^{5}$ White solid; m.p. 163-164 ${ }^{\circ} \mathrm{C} ;{ }^{1} \mathrm{H}$ NMR (300 MHz, Chloroform- $d$ ) 7.02 - $7.10(\mathrm{~m}, 1 \mathrm{H}), 7.37-7.48(\mathrm{~d}, 2 \mathrm{H}), 7.87-8.27$ (m, 5H), $8.45-8.49$ (d, 2H), $9.89-9.98(\mathrm{~s}, 1 \mathrm{H})$. ${ }^{13} \mathrm{C}$ NMR (125 MHz, Chloroform-d): $\delta$ 120.32, 123.93, 126.56, 128.68, 128.93, 131.99, 135.61, $138.70,166.03$.<smiles>O=C(Nc1ccccc1)c1ccccc1</smiles>

9 4-methoxy-N-phenylbenzamide:5 White solid, m.p: 170-171 ${ }^{\circ} \mathrm{C},{ }^{1} \mathrm{H}$ NMR $(300 \mathrm{MHz}$, Chloroform- $d$ ) $\delta 4.22-4.30(\mathrm{~s}, 3 \mathrm{H}), 7.12-7.24(\mathrm{~d}, 2 \mathrm{H}), 7.38-7.60(\mathrm{~m}, 5 \mathrm{H}), 7.90-7.98(\mathrm{~d}, 2 \mathrm{H})$, $10.59-10.61(\mathrm{~s}, 1 \mathrm{H})$. 
<smiles>COc1ccc(C(=O)Nc2ccccc2)cc1</smiles>

10 N-(4-methoxyphenyl)benzamide: ${ }^{6}$ Yellow solid; m.p. 157-158 ${ }^{\circ} \mathrm{C},{ }^{1} \mathrm{H}$ NMR $(300 \mathrm{MHz}$, Chloroform- $d$ ) $\delta 2.15-2.34(\mathrm{~s}, 3 \mathrm{H}), 7.17-7.36(\mathrm{~d}, 2 \mathrm{H}), 7.39-7.59(\mathrm{~m}, 5 \mathrm{H}), 7.78-7.90(\mathrm{~d}, 2 \mathrm{H})$, $9.98-10.01(\mathrm{~s}, 1 \mathrm{H}),{ }^{13} \mathrm{C}$ NMR (125 MHz, Chloroform-d): $\delta$ 52.9, 112.5, 116.9, 122.3, 126.5, $127.5,128.3,138.6,159.4,163.8$.<smiles>COc1ccc(NC(=O)c2ccccc2)cc1</smiles>

11 N-(p-tolyl)benzamide: ${ }^{7}$ Yellow solid; m.p. $157-158{ }^{\circ} \mathrm{C}$, mass spectroscopy: 211.10 , found: 211.9.<smiles>Cc1ccc(NC(=O)c2ccccc2)cc1</smiles>

12 4-bromo-N-phenylbenzamide: ${ }^{8}$ White solid, m.p. 133-134 ${ }^{\circ} \mathrm{C}, 1 \mathrm{H}$ NMR $(300 \mathrm{MHz}$, Chloroform-d) $\delta 6.05-6.21(\mathrm{~m}, 1 \mathrm{H}), 6.44-6.53(\mathrm{~m}, 2 \mathrm{H}), 7.49-7.50(\mathrm{~m}, 4 \mathrm{H}), 7.74-7.83(\mathrm{~d}$, $2 \mathrm{H}), 9.85-9.88(\mathrm{~s}, 1 \mathrm{H})$.<smiles>O=C(Nc1ccccc1)c1ccc(Br)cc1</smiles> 
13 4-fluoro-N-(p-tolyl)benzamide: ${ }^{9}$ White solid, m.p. 202-210 ${ }^{\circ}$ C, ${ }^{1} \mathrm{H}$ NMR $(300 \mathrm{MHz}$, Chloroform-d) $\delta 2.38(\mathrm{~s}, 3 \mathrm{H}), 7.11(\mathrm{t}, 2 \mathrm{H}), 7.30(\mathrm{~d}, 2 \mathrm{H}), 7.83-7.91(\mathrm{~m}, 4 \mathrm{H}), 9.55(\mathrm{~s}, 1 \mathrm{H})$.

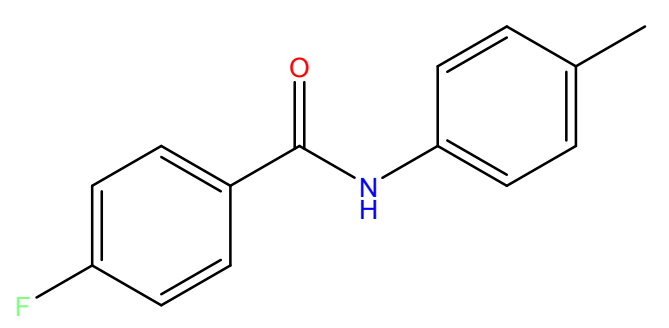

14 N-(4-fluorophenyl) benzamide: ${ }^{10}$ White solid, m.p. $167-170{ }^{\circ} \mathrm{C}$; ${ }^{1} \mathrm{H}$ NMR $(300 \mathrm{MHz}$, Chloroform-d) $\delta 7.01(\mathrm{t}, 1 \mathrm{H}), 7.35(\mathrm{~d}, 2 \mathrm{H}), 7.78(\mathrm{~d}, 2 \mathrm{H}), 7.65(\mathrm{~d}, 2 \mathrm{H}), 8.11(\mathrm{~d}, 2 \mathrm{H}), 9.55(\mathrm{~s}$, $1 \mathrm{H})$.<smiles>O=C(Nc1ccc(F)cc1)c1ccccc1</smiles> 


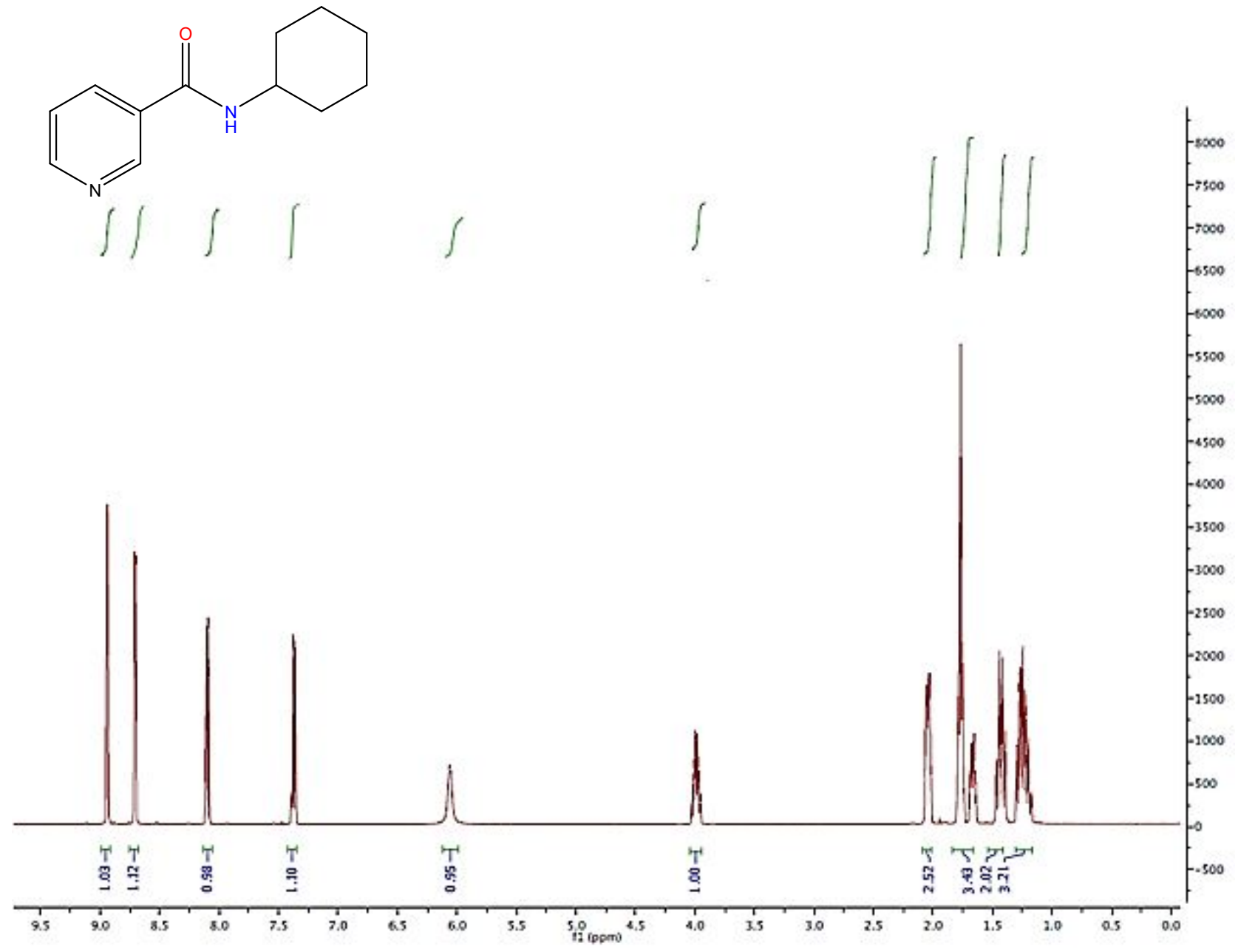

Figure S1 ${ }^{1}$ HNMR N-Cyclohexylnicotinamide 


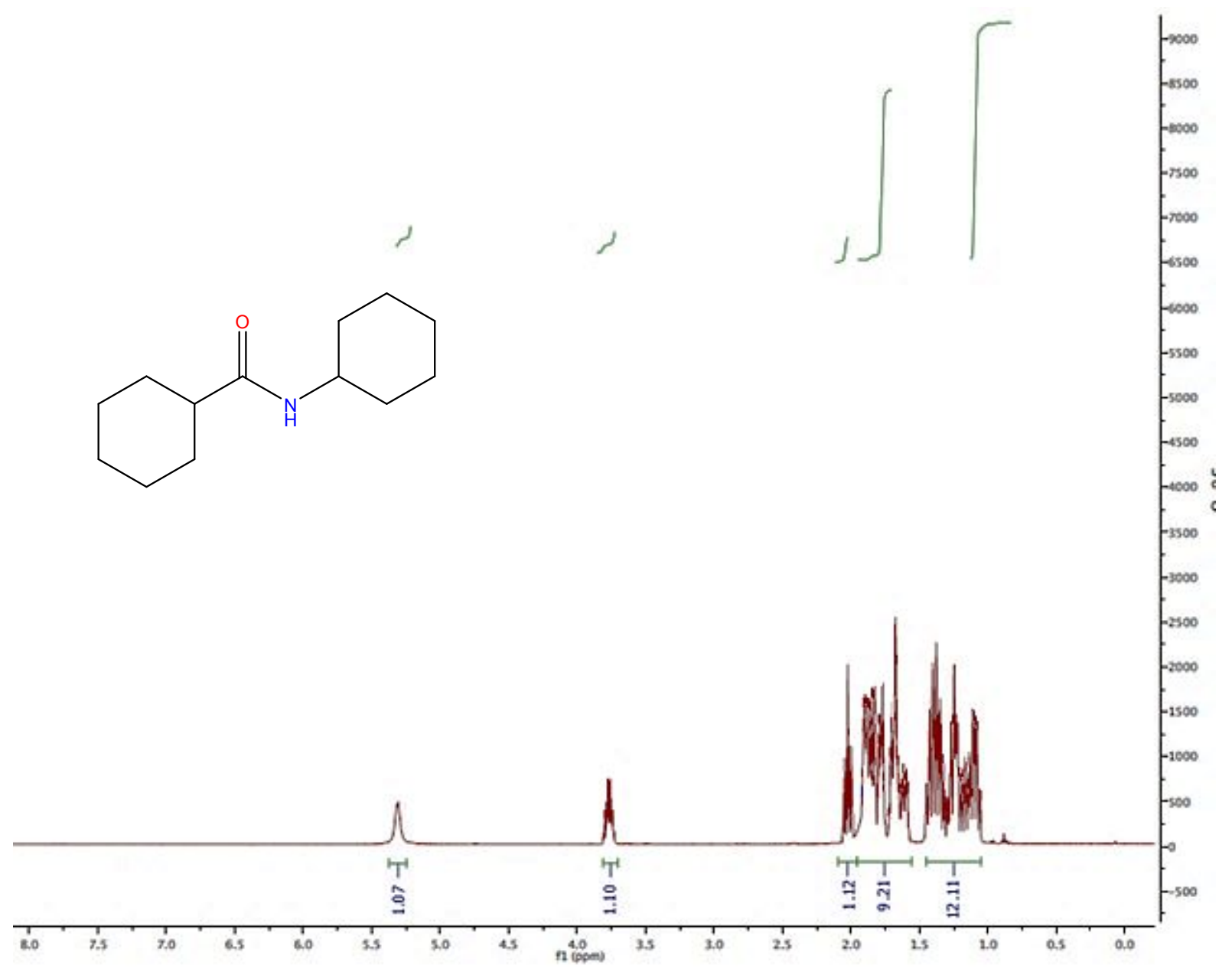

Figure S2 ${ }^{1} \mathrm{HNMR}$ N-cyclohexylcyclohexanecarboxamide 


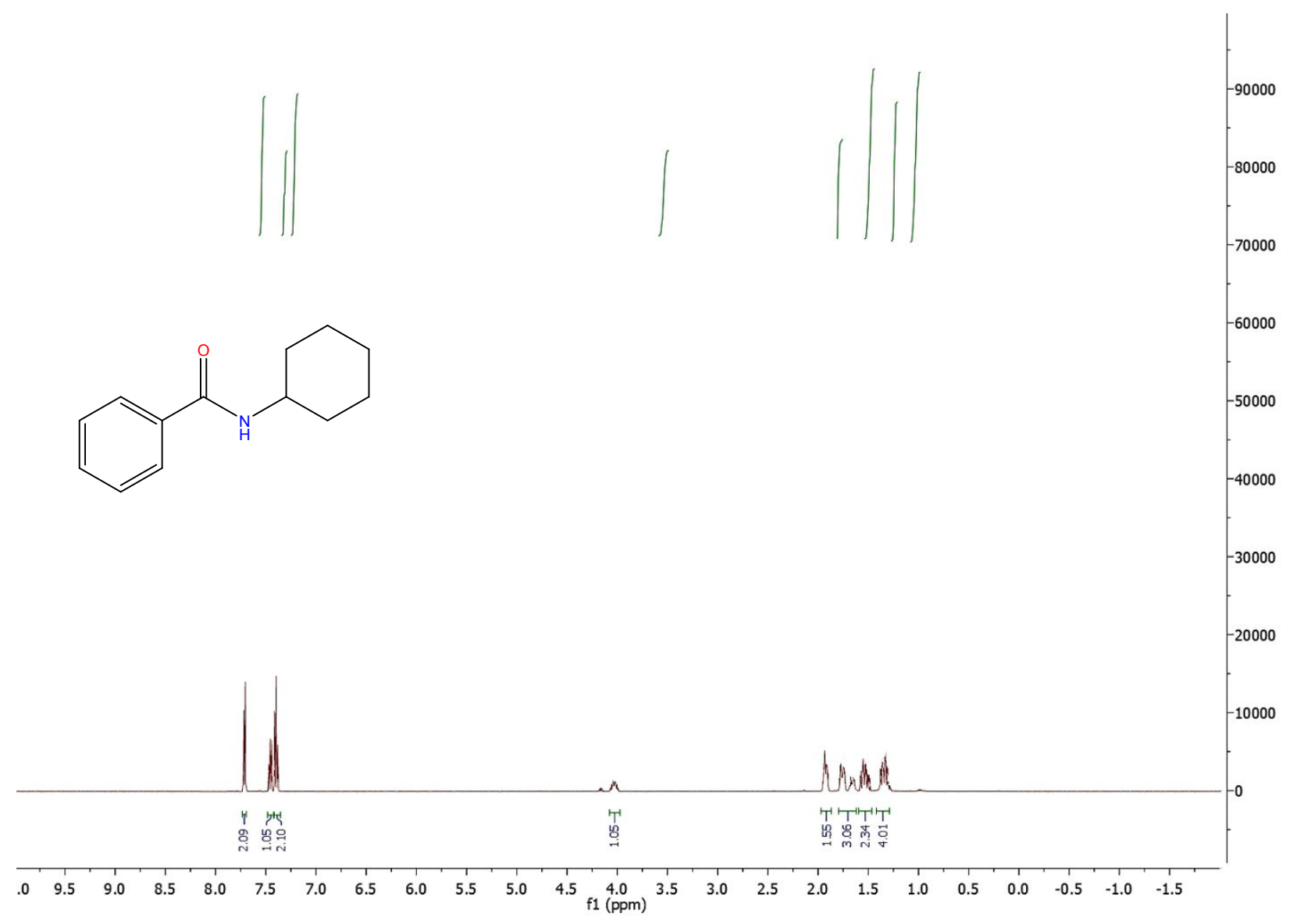

Figure S3 ${ }^{1} \mathrm{HNMR}$ N-cyclohexylbenzamide 


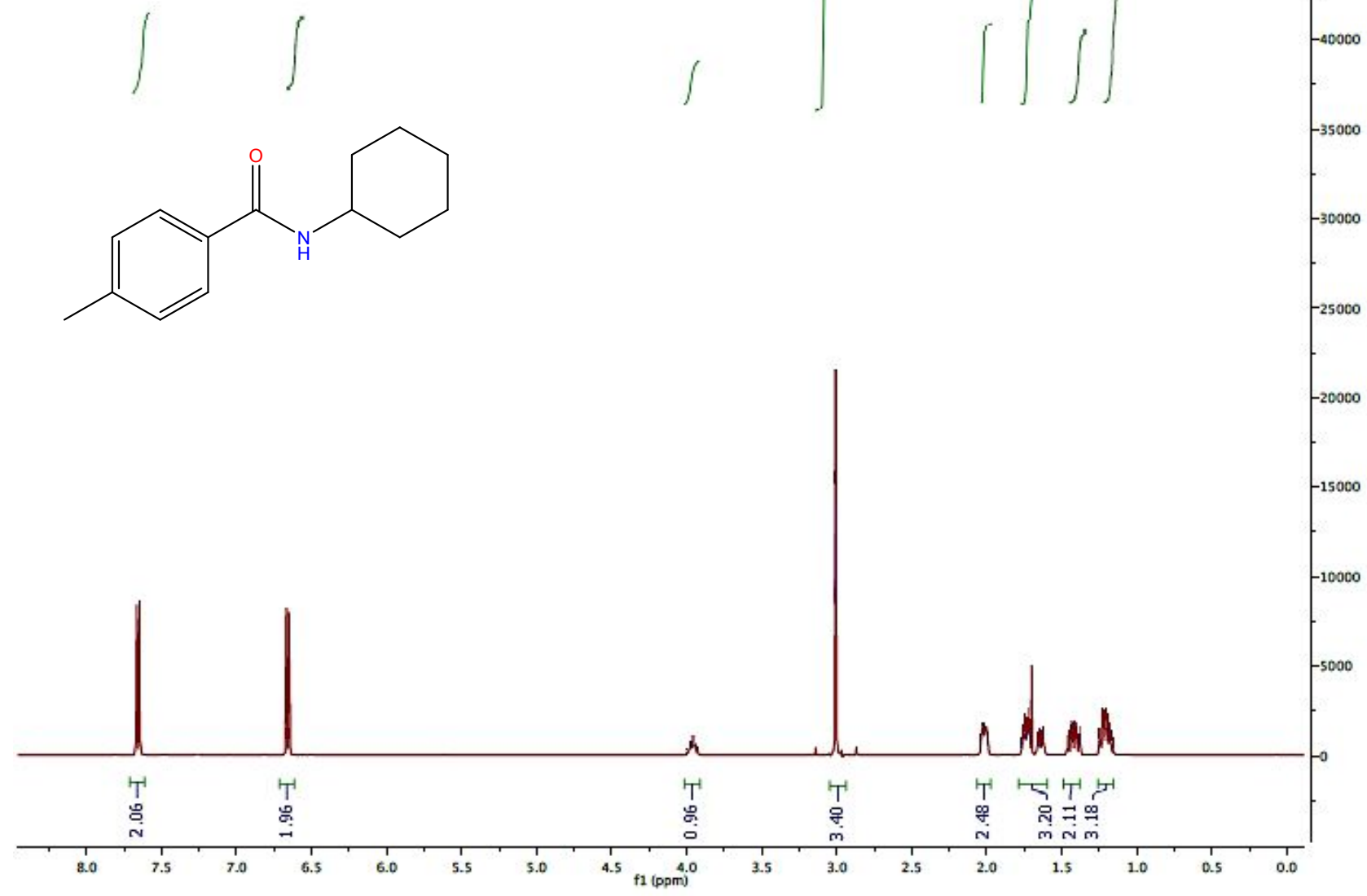

Figure S4 ${ }^{1} \mathrm{HNMR}$ N-cyclohexyl-4-methylbenzamide 


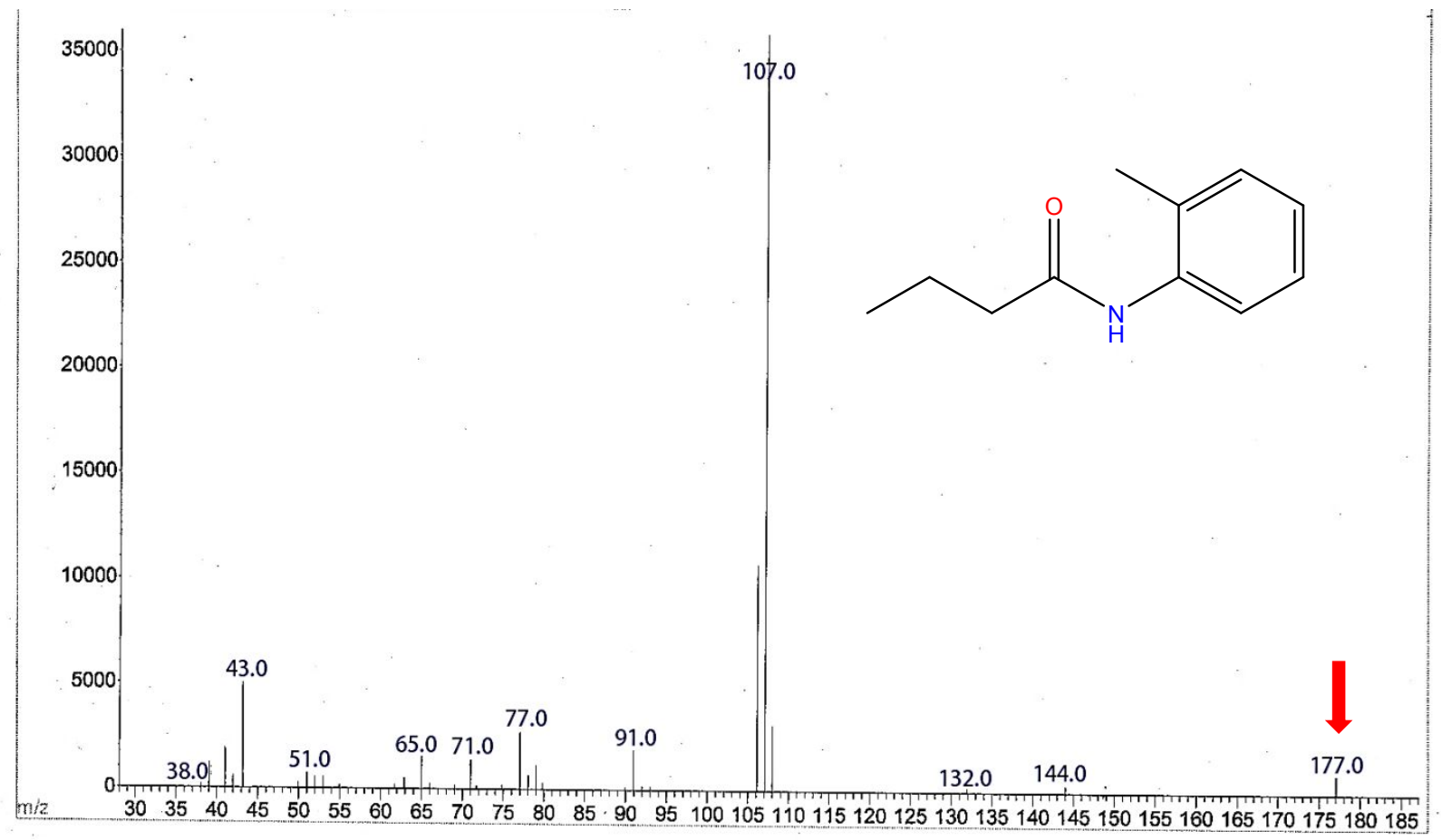

Figure S5 mass N-(o-tolyl)butyramide 


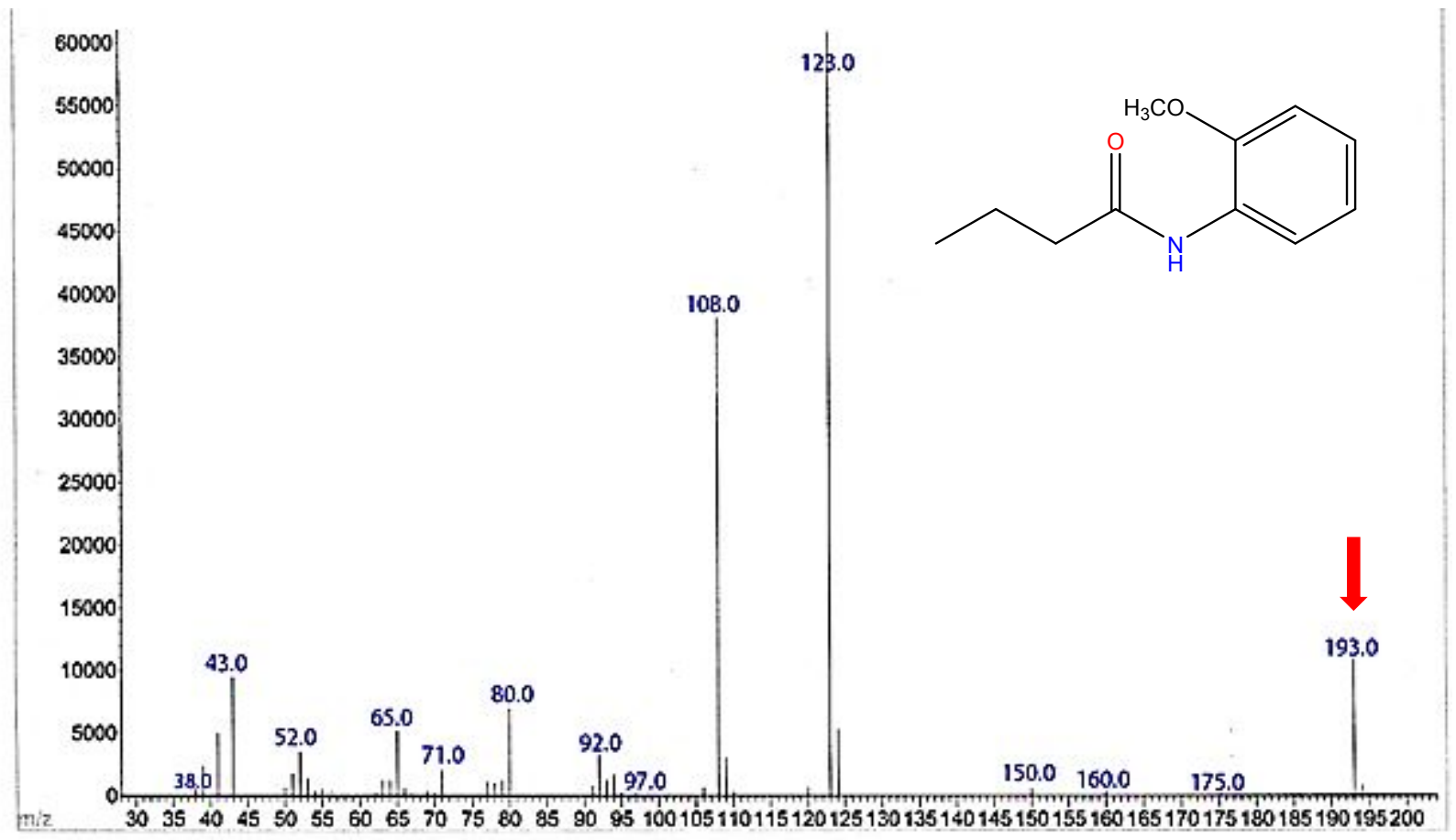

Figure S6 mass N-phenylbutyramide 


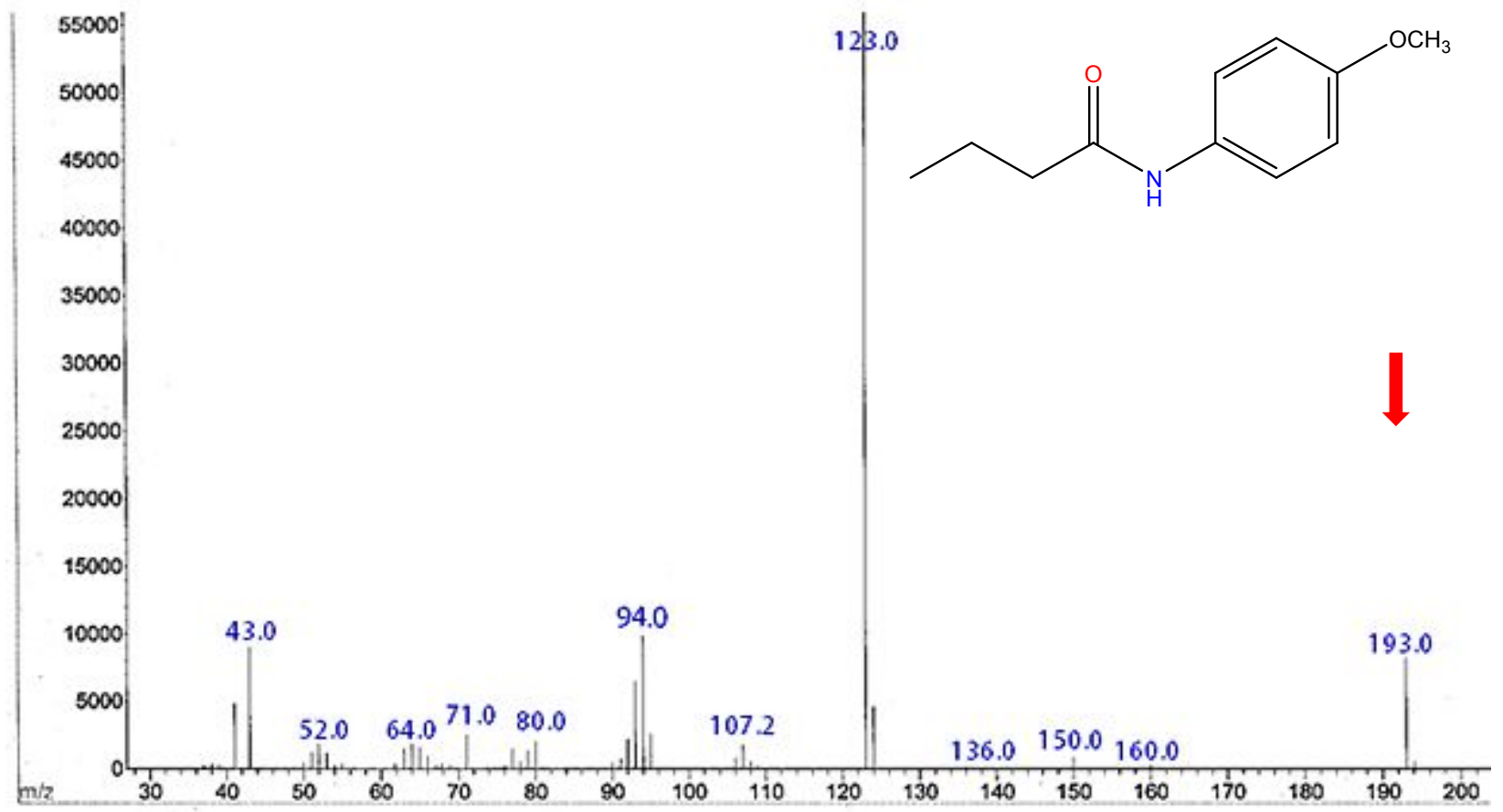

Figure S7 mass N-(4-methoxyphenyl)propionamide 


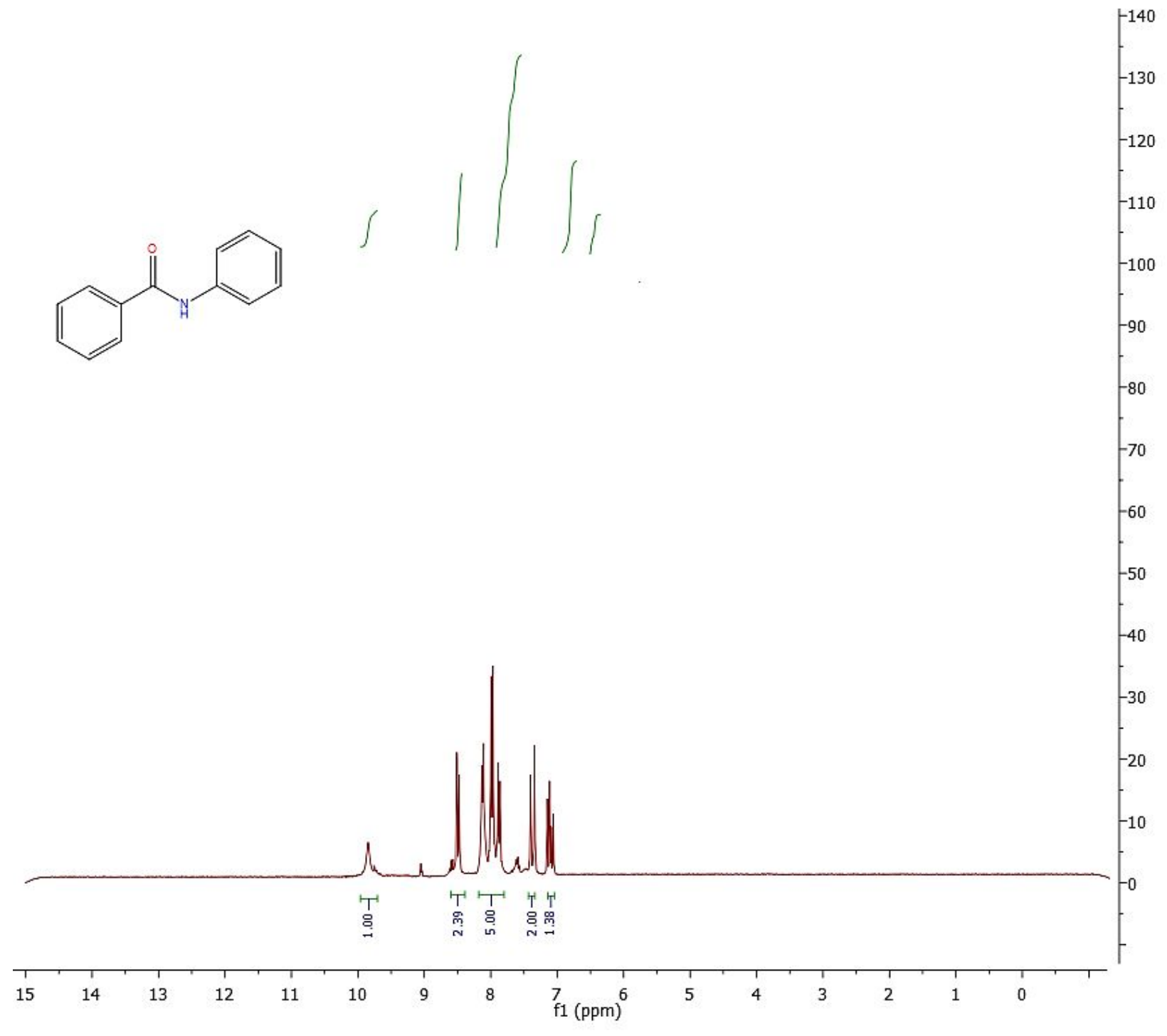

Figure S8 ${ }^{1}$ HNMR N-phenylbenzamide 


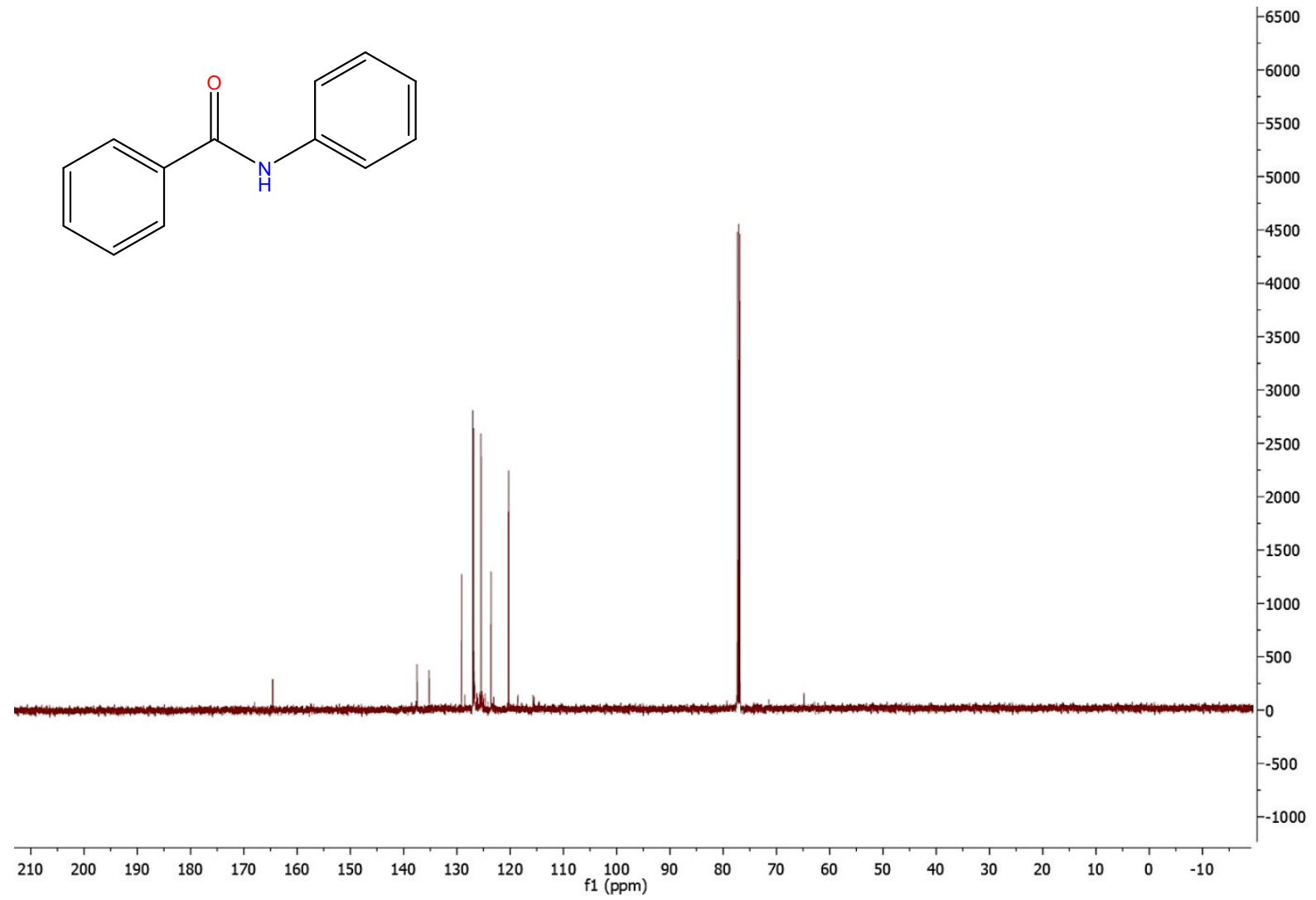

Figure S9 ${ }^{13} \mathrm{CNMR} \mathbf{N}$-phenylbenzamide 


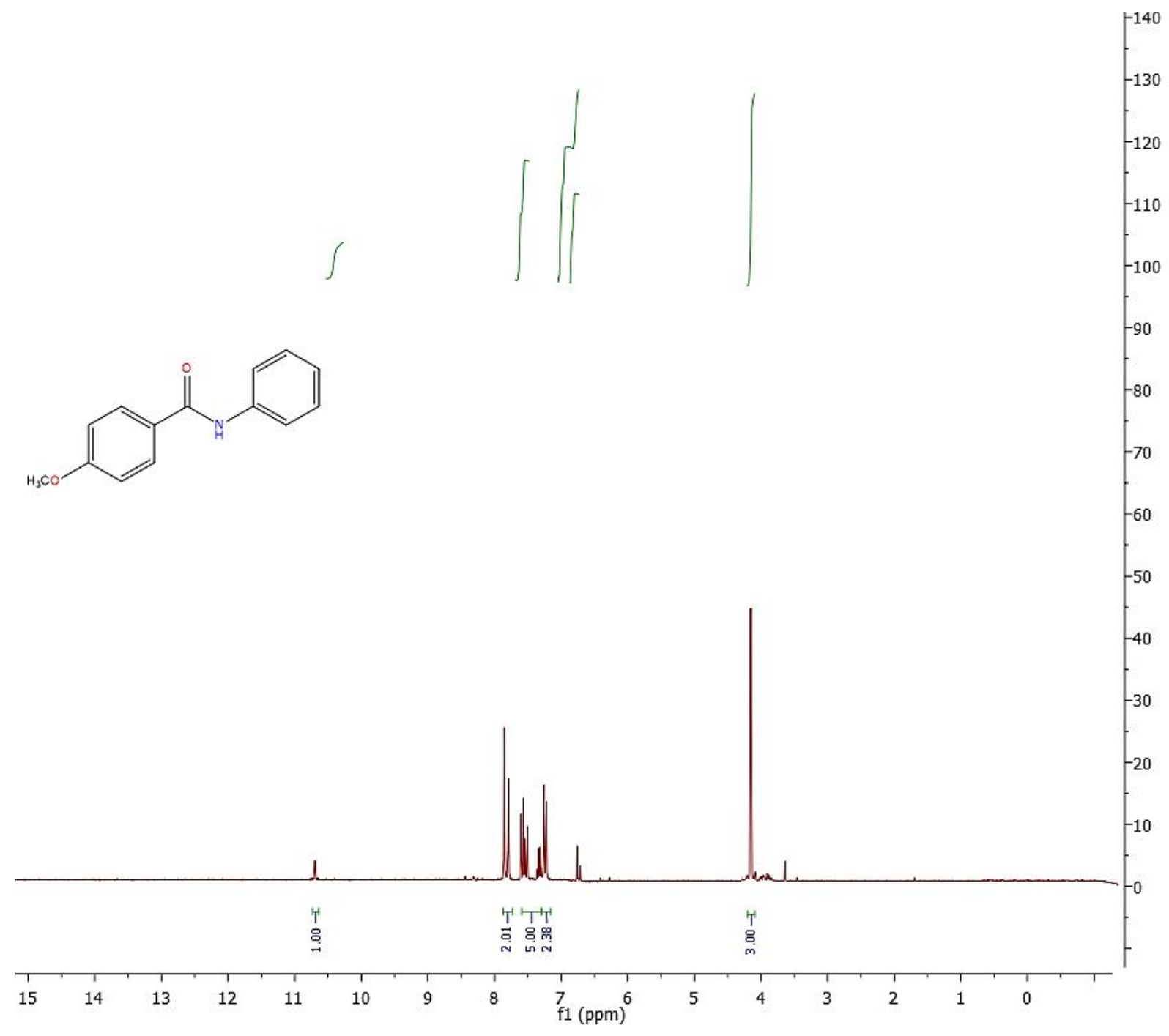

Figure S10 ${ }^{1}$ HNMR 4-methoxy-N-phenylbenzamide 


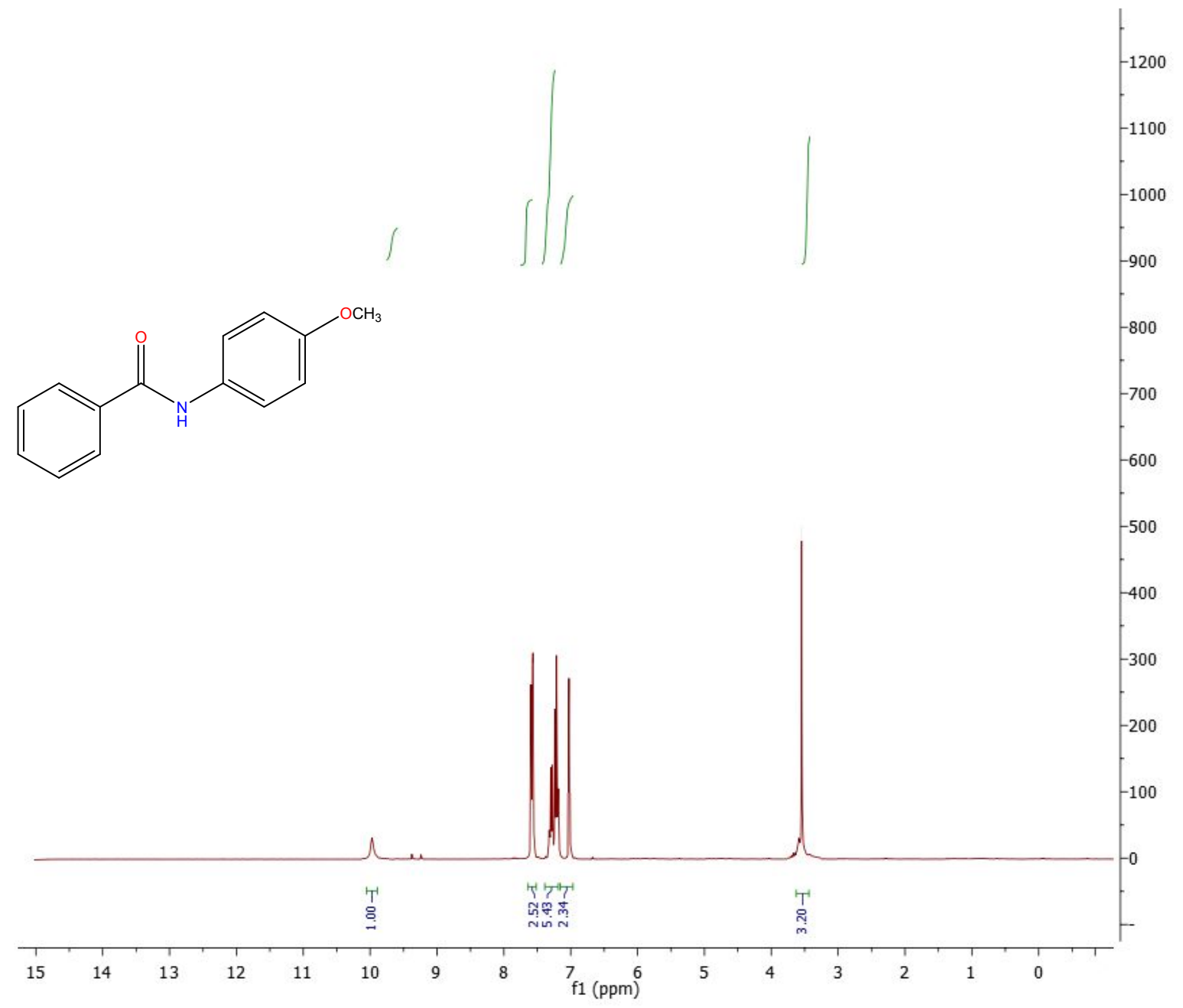

Figure S1 $1{ }^{1} \mathrm{HNMR}$ N-(4-methoxyphenyl)benzamide 


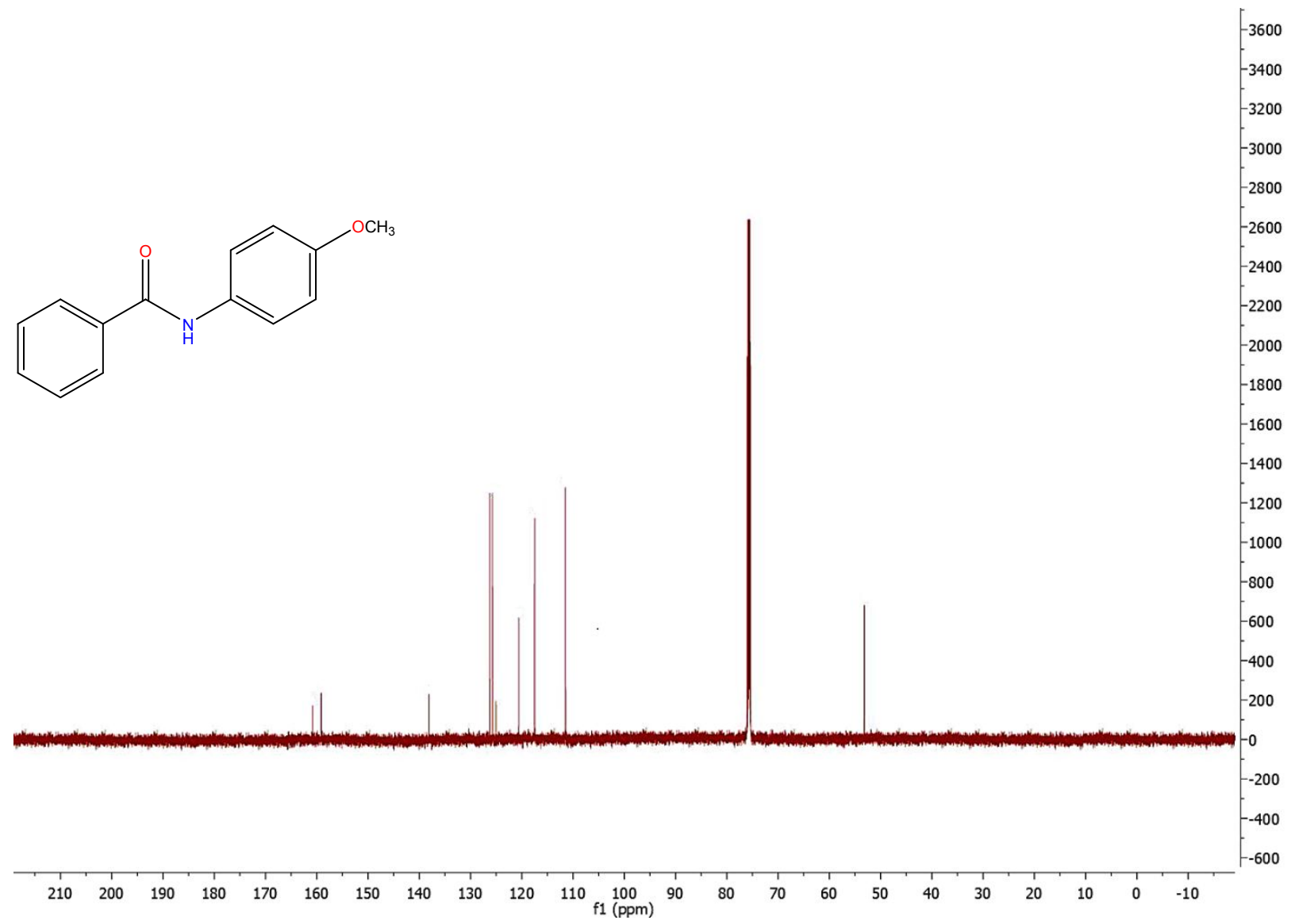

Figure S12 ${ }^{13} \mathrm{CNMR}$ N-(4-methoxyphenyl)benzamide 


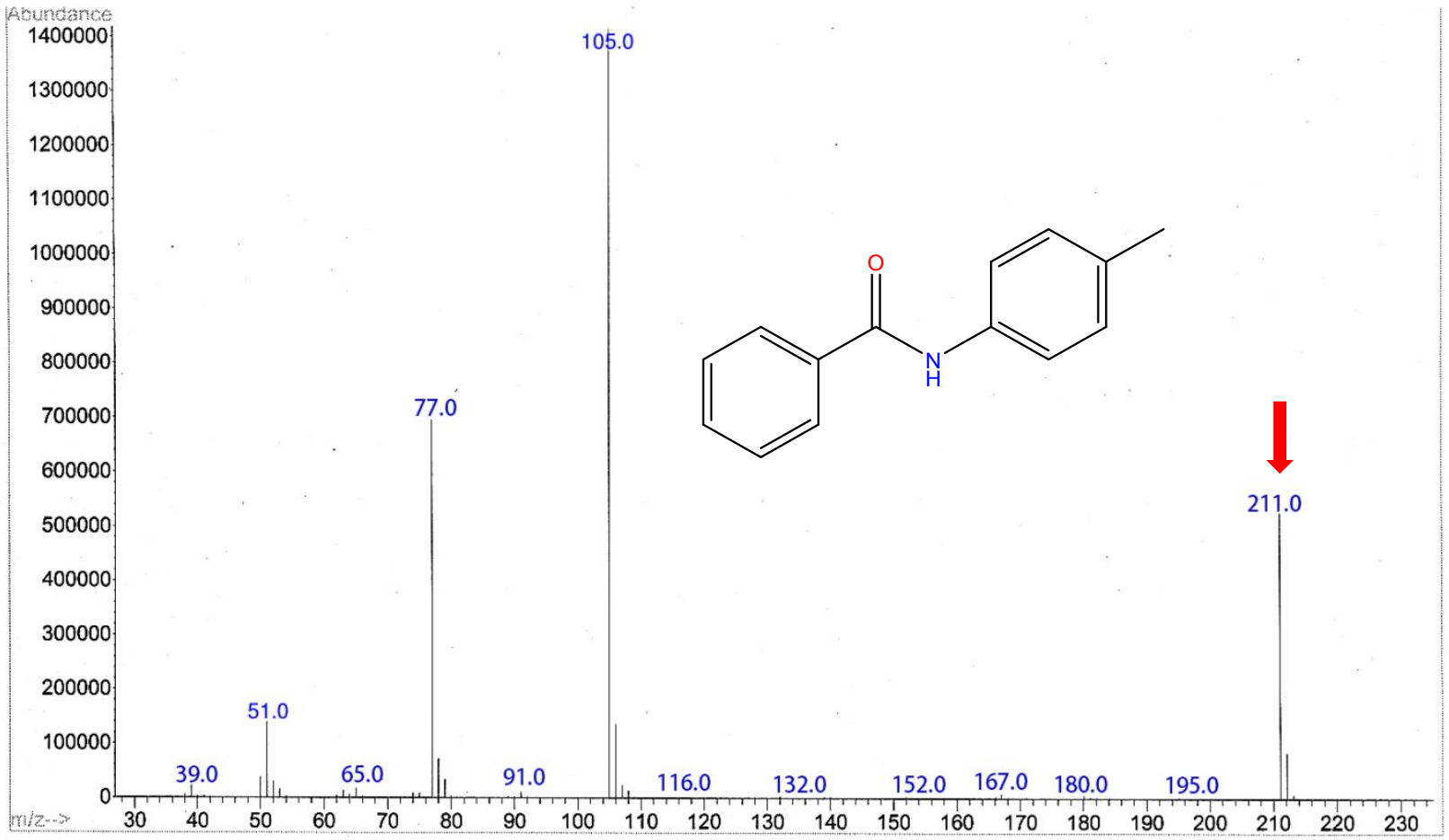

Figure S13 mass N-(p-tolyl)benzamide 
<smiles>O=C(Nc1ccccc1)c1ccc(Br)cc1</smiles>

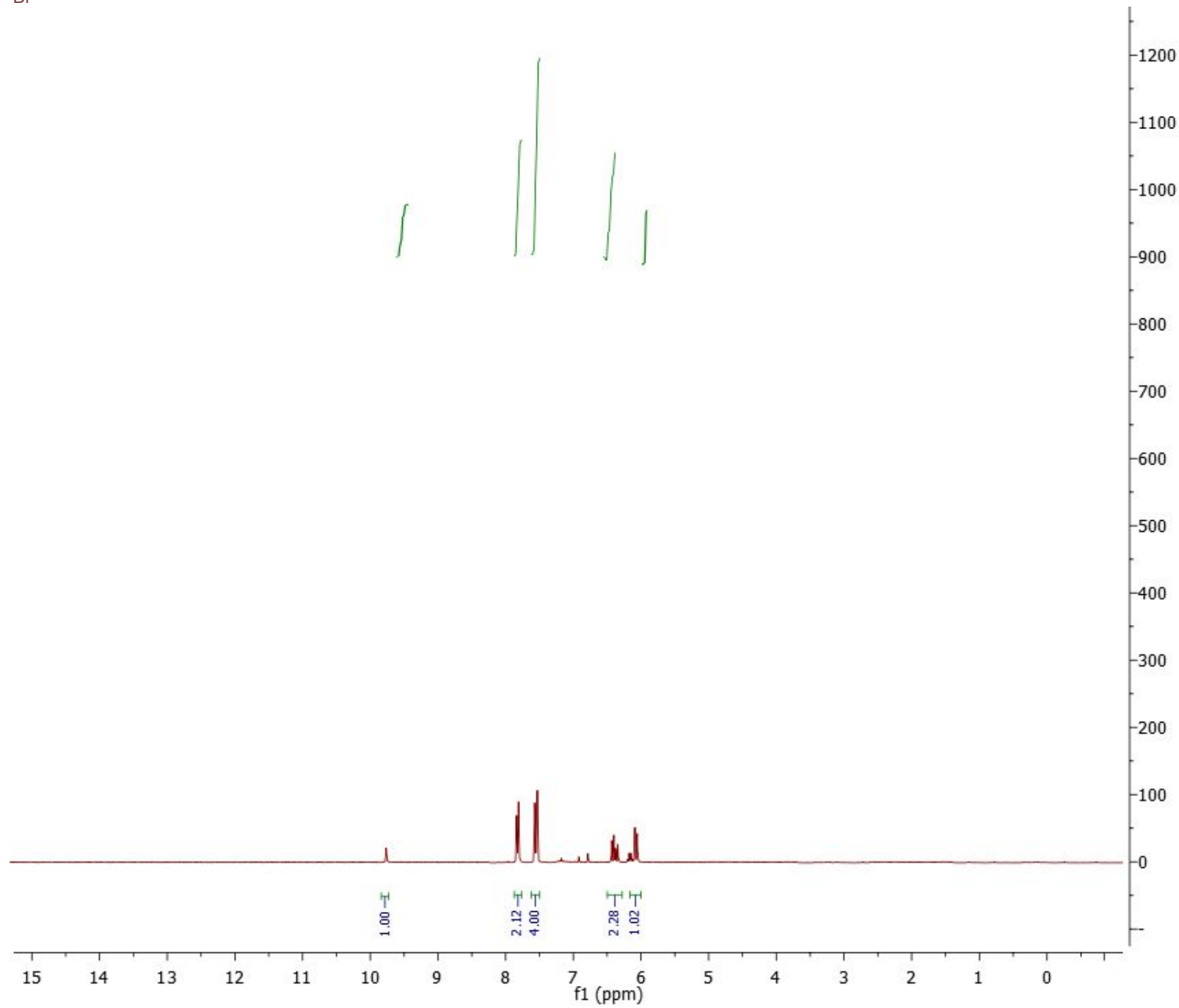

Figure S14 ${ }^{1}$ HNMR 4-bromo-N-phenylbenzamide 


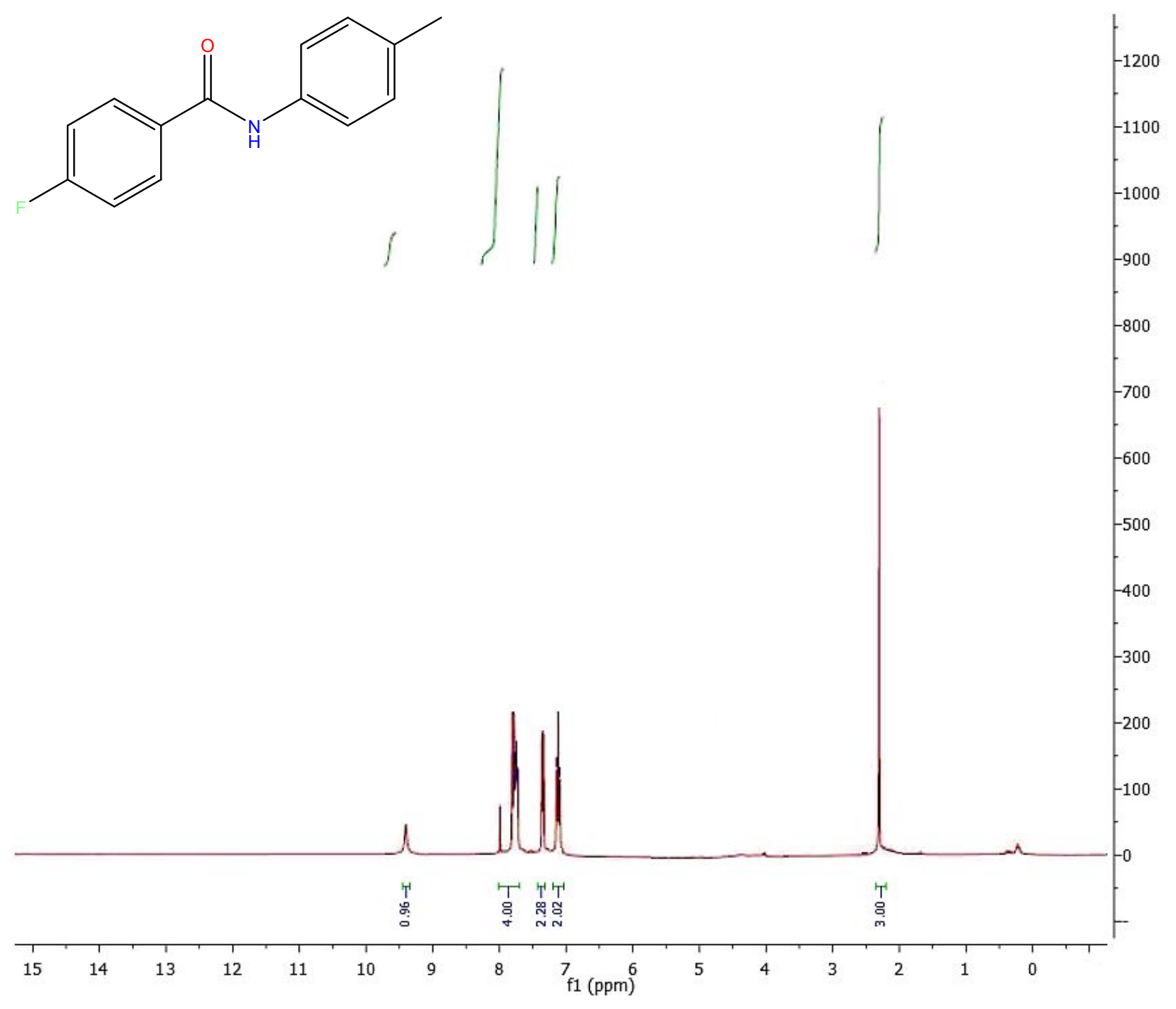

Figure S15 ${ }^{1} \mathrm{HNMR}$ 4-fluoro-N-(p-tolyl)benzamide 


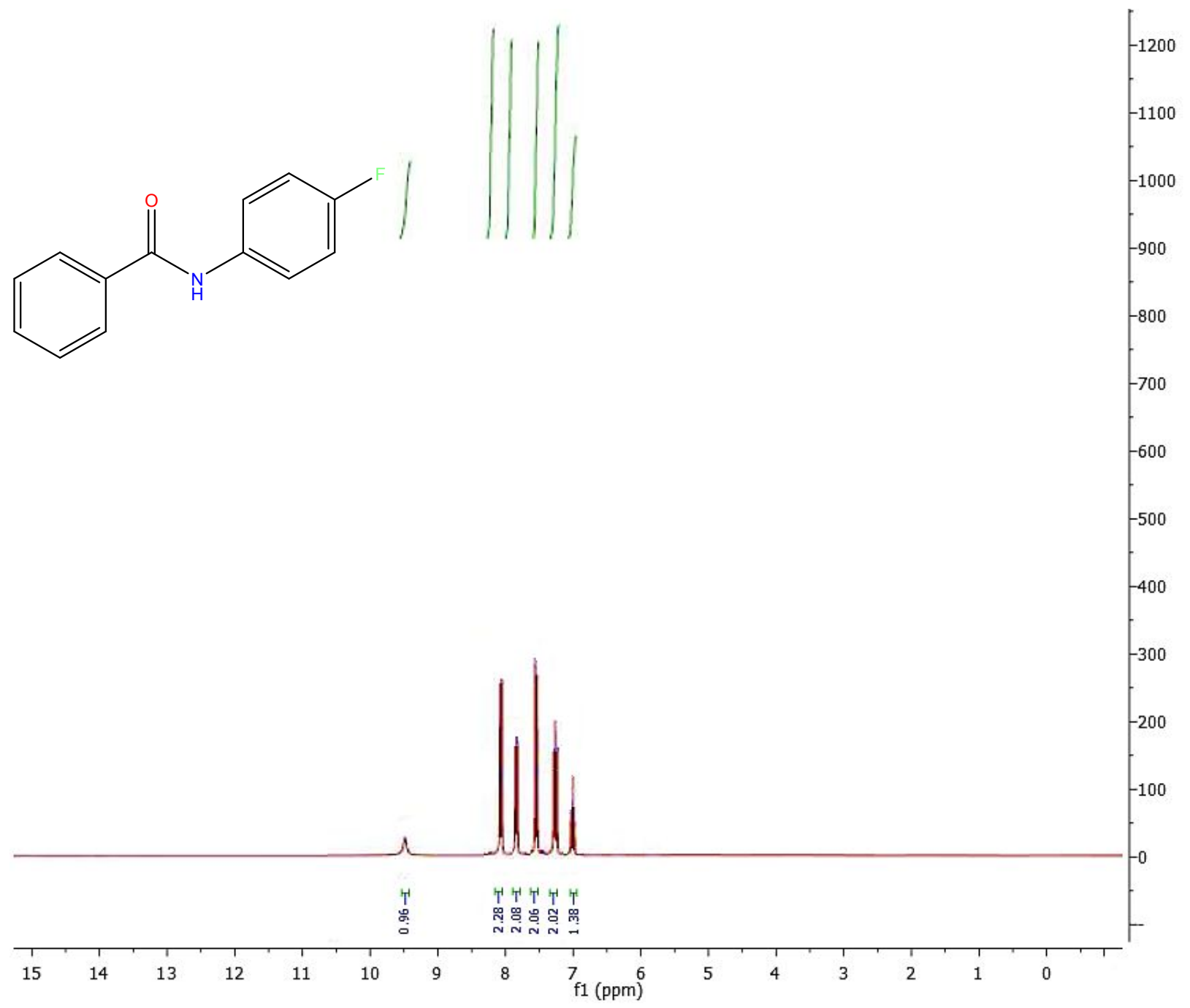

Figure S16 ${ }^{1} \mathrm{HNMR}$ N-(4-fluorophenyl) benzamide

\section{References}


(1) Auzeil, N.; Largeron, M.; Fleury, M.-B. Acid-Catalysed N-Alkyl Heterolysis of Tertiary Pyridinecarboxamides and Benzamides under Mild Conditions. J. Chem. Soc. Perkin Trans. 2 1999, No. 8, 1703-1710.

(2) Chow, S. Y.; Stevens, M. Y.; Åkerbladh, L.; Bergman, S.; Odell, L. R. Mild and Low-Pressure Fac-Ir (Ppy) 3-Mediated Radical Aminocarbonylation of Unactivated Alkyl Iodides through Visible-Light Photoredox Catalysis. Chem. Eur. J. 2016, 22 (27), 91559161.

(3) Gockel, S. N.; Hull, K. L. Chloroform as a Carbon Monoxide Precursor: In or Ex Situ Generation of CO for Pd-Catalyzed Aminocarbonylations. Org. Lett. 2015, 17 (13), 32363239.

(4) $\mathrm{Tu}, \mathrm{Y}$. Fragmentation of Conjugated Amides at the $\mathrm{C} \square \mathrm{C}(\mathrm{O})$ Bond in Electrospray Mass Spectrometry: A Proton-bound Dimeric Intermediate Identified by the Kinetic Method. Rapid Commun. mass Spectrom. 2004, 18 (12), 1345-1351.

(5) Swamy, T.; Raviteja, P.; Subba Reddy, B. V; Ravinder, V. Efficient Method for the Synthesis of Benzamides from Benzoic Acids and Aryl Isothiocyanates Using K2HPO4. ChemistrySelect 2017, 2 (25), 7612-7614.

(6) Ding, Y.; Zhang, X.; Zhang, D.; Chen, Y.; Wu, Z.; Wang, P.; Xue, W.; Song, B.; Yang, S. Copper-Catalyzed Oxidative Amidation between Aldehydes and Arylamines under Mild Conditions. Tetrahedron Lett. 2015, 56 (6), 831-833.

(7) Deng, L.; Huang, B.; Liu, Y. Copper (Ii)-Mediated, Carbon Degradation-Based Amidation of Phenylacetic Acids toward N-Substituted Benzamides. Org. Biomol. Chem. 2018, 16 (9), $1552-1556$.

(8) Tan, Z.; Li, Z.; Ma, Y.; Qin, J.; Yu, C. Potassium Tert-Butoxide Prompted Highly Efficient Transamidation and Its Coordination Radical Mechanism. European J. Org. Chem. 2019, 2019 (28), 4538-4545.

(9) Bose, D. S.; Idrees, M.; Todewale, I. K.; Jakka, N. M.; Rao, J. V. Hybrids of Privileged Structures Benzothiazoles and Pyrrolo [2, 1-c][1, 4] Benzodiazepin-5-One, and DiversityOriented Synthesis of Benzothiazoles. Eur. J. Med. Chem. 2012, 50, $27-38$.

(10) Xu, M.; Zhang, X.; Shao, Y.; Han, J.; Zhong, P. The Synthesis of N-Arylated Amides via Copper (II) Triflate-Catalyzed Direct Oxygenation and N-Arylation of Benzylamines with Aryl Iodides. Adv. Synth. Catal. 2012, 354 (14-15), 2665-2670. 